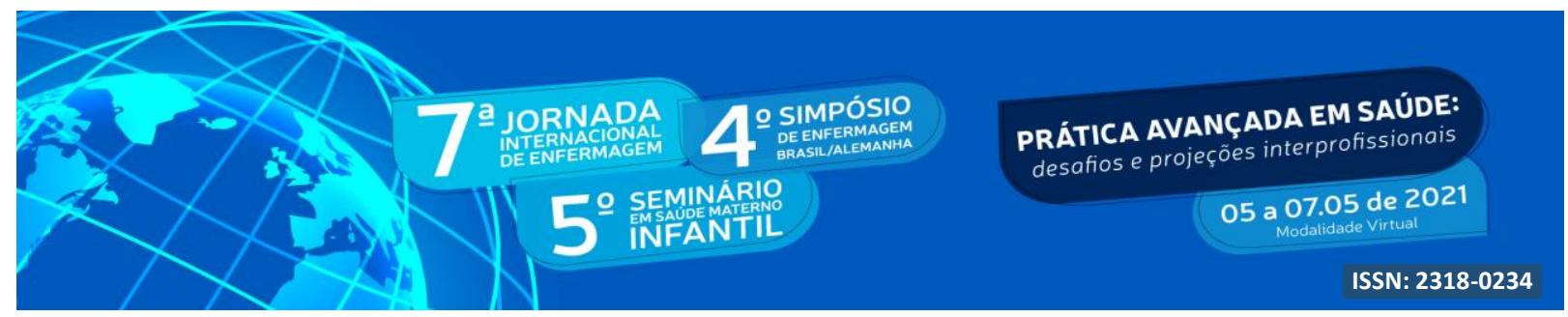

DOI: http://doi.org/10.48195/jie2021-064

\title{
A ENFERMAGEM NA PERSPECTIVA DE ESTUDANTES DE GRADUAÇÃO SOBRE O TESTE DE GUTHRIE: REVISÃO NARRATIVA ${ }^{1}$
}

\author{
Adriélli Balconi $^{2}$; Alielly Camargo ${ }^{3}$; Anna Júlia Pacheco ${ }^{4}$; Isadora Balconi ${ }^{5}$; Isadora \\ Vasconcelos $^{6}$; Priscila Kruz ${ }^{7}$
}

\begin{abstract}
RESUMO
O presente estudo objetiva refletir acerca da relevância na realização precoce do teste do pezinho e a necessidade da qualificação por parte dos profissionais de enfermagem para orientar os pais e/ou responsáveis sobre a importância do teste para o recém-nascido. Trata-se de uma revisão bibliográfica do tipo narrativa com abordagem qualitativa. Os resultados revelam que os responsáveis dos RNS desconhecem as doenças identificadas no exame e a sua significância para a qualidade de vida. Na vivência profissional, tem se observado que muitos enfermeiros não detém conhecimento técnicocientífico para instruir e coletar o exame. Desta forma, a capacitação de profissionais de saúde em relação ao cuidado, torna-se fundamental para prestar assistência de qualidade, a fim de orientar os pais sobre as patologias detectadas na Triagem Neonatal.
\end{abstract}

Palavras-chave: Cuidados de Enfermagem; Recém-Nascido; Saúde Materno-Infantil; Teste de Guthrie.

\begin{abstract}
The present study aims to reflect on the relevance in the early performance of the heel prick test and the need for qualification on the part of nursing professionals to guide parents and / or guardians about the importance of the test for the newborn. It is a bibliographic review of the narrative type with a qualitative approach. The results reveal that those responsible for the RNS are unaware of the diseases identified in the examination and their significance for quality of life. In professional experience, it has been observed that many nurses do not have technical-scientific knowledge to instruct and collect the exam. Thus, the training of health professionals in relation to care becomes essential to provide quality care, in order to guide parents about the pathologies detected in Neonatal Screening.
\end{abstract}

Key Words: Nursing Care; Newborn; Maternal and Child Health; Guthrie test.

\footnotetext{
${ }^{1}$ Iniciação Científica. Santa Maria. RS.

${ }^{2}$ Estudante do Curso de Enfermagem. FISMA. Santa Maria. RS. E-mail: adriidalgobalconi@gmail.com

${ }^{3}$ Estudante do Curso de Enfermagem. URCAMP. Bagé. RS. E-mail: aliellycamargo2504@gmail.com

${ }^{4}$ Estudante do Curso de Enfermagem. UFSM. Santa Maria. RS. E-mail: anna.lilo2000@ gmail.com

${ }^{5}$ Estudante do Curso de Enfermagem. UFSM. Santa Maria. RS. E-mail: isadorapbalconi@gmail.com

${ }^{6}$ Estudante do Curso de Enfermagem. URCAMP. Bagé. RS. E-mail: amaralvasconcelosisadora@gmail.com

${ }^{7}$ Orientadora. Enfermeira. Professora. FISMA. Santa Maria. RS. E-mail: priscila.kurz@ fisma.com.br
} 


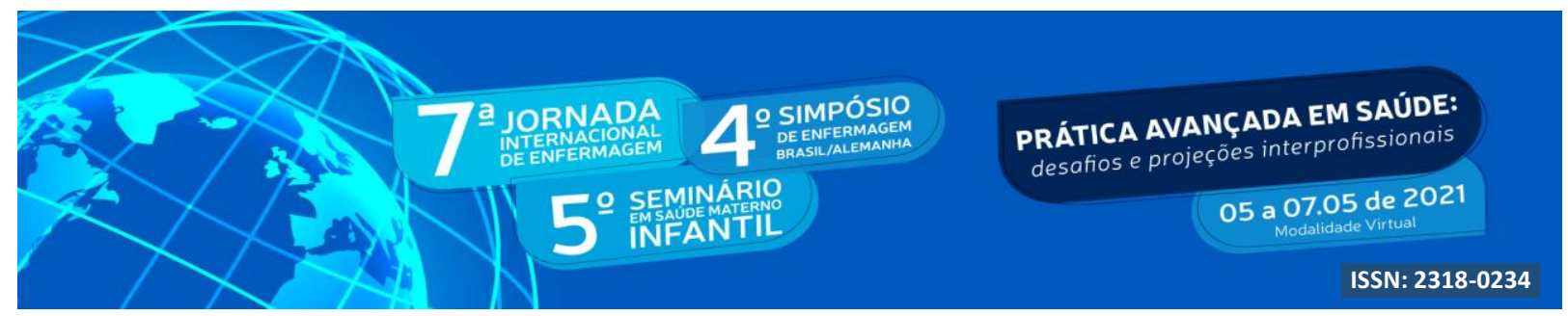

\section{INTRODUÇÃO}

O Teste de Guthrie ou Teste do Pezinho, faz parte da Triagem Neonatal (TN), juntamente a outros testes. Trata-se de um exame laboratorial, por meio da coleta de sangue no calcanhar dos recém-nascidos $(\mathrm{RN})$, realizado para a identificação precoce de doenças genéticas, metabólicas e infecciosas. Estas, quando tratadas precocemente permitem o desenvolvimento físico e mental adequados. Realiza-se, preferencialmente, entre o $3^{\circ}$ e $5^{\circ}$ dias de vida e sempre após 48 horas da primeira amamentação do RN. Além disso, esse teste pode ser realizado no prazo máximo de 30 dias, no entanto, quanto antes realizado, mais precoce será o tratamento em pacientes afetados BRASIL (2019a).

Tendo em vista a prevenção de deficiências mentais e agravos à saúde do RN, a Organização Mundial da Saúde (OMS) recomenda a implantação de programas nacionais de TN. Dessa forma, esta foi incorporada ao Sistema Único de Saúde (SUS), em janeiro de 1992, por meio do Teste do Pezinho (TP), a qual tornava obrigatório a realização deste em todos os recém-nascidos para a avaliação de Fenilcetonúria e Hipotireoidismo Congênito. Desde então, configura-se um exame obrigatório e ofertado pela rede pública de saúde, dessa forma, embora as doenças detectadas não possuam cura, o diagnóstico e tratamento precoce propiciam um bom prognóstico BRASIL (2002).

Nesse ínterim, por meio da Portaria n. ${ }^{\circ}$ 822, de 6 de junho de 2001, o Ministério da Saúde criou o Programa Nacional de Triagem Neonatal (PNTN), com o intuito de ampliar as patologias triadas para além da Fenilcetonúria e Hipotireiodismo Congênito, passando a abranger, com a última reformulação, a Doença falciforme e outras hemoglobinopatias, Fibrose Cística, Hiperplasia Adrenal e Congênita e Deficiência de Biotinidase BRASIL (2002).

Com a criação do PNTN, objetivou-se atingir a cobertura de $100 \%$ do público-alvo, com a busca ativa, confirmação de diagnóstico e a oferta de um cuidado multiprofissional, reduzindo a morbimortalidade. Todavia, conforme estimativas recentes, cerca de $80 \%$ das 


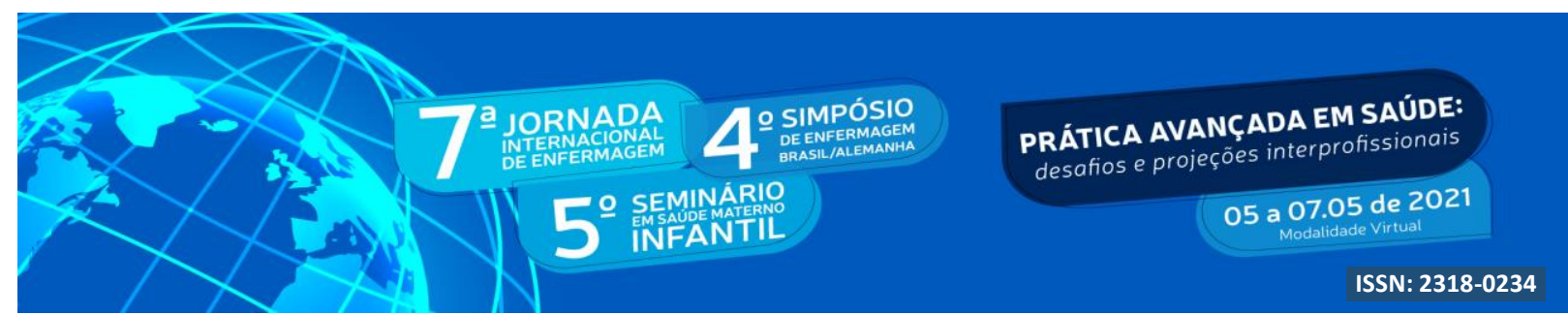

crianças nascidas no país realizam o TP no SUS BRASIL (2018).

Nesse sentido, frente às lacunas que envolvem a realização dos testes no país, compreende-se a relevância de tal exame e do tratamento precoce à qualidade de vida. Por isso, os profissionais que atuam diretamente com gestantes, parturientes, puérperas e RN necessitam do conhecimento sobre os testes a serem realizados, a idade indicada, as doenças que são identificadas, os benefícios da detecção precoce, a fim de orientar previamente os pais e a família, para alcançar $100 \%$ de cobertura. Isso evidencia o exercício profissional da equipe de enfermagem, pois dispõe da atuação em diferentes cenários com o público-alvo, desde a Atenção Primária à Saúde (APS) à Atenção Hospitalar.

Embora a realização do teste do pezinho seja de competência do profissional de Enfermagem, muitos ainda carecem de capacitação técnica científica para a coleta, registro e armazenamento adequado das amostras, assim como a orientação sobre a importância desse exame para evitar insegurança e evasão (MARQUI, 2016). Dessa forma, mostra-se relevante discutir sobre a atuação desses profissionais na prevenção de agravos pela execução qualificada da Triagem Neonatal.

\section{OBJETIVO}

Apresentar a relevância da realização precoce do Teste do Pezinho e a necessidade da qualificação por parte dos profissionais de enfermagem.

\section{METODOLOGIA}

Trata-se de uma revisão bibliográfica do tipo narrativa com abordagem qualitativa. Conforme Gil (2017), a pesquisa bibliográfica constitui-se a partir de materiais já publicados, incluindo livros, revistas e artigos científicos. Além disso, o estudo do tipo narrativo permite uma abrangência, buscando identificar a temática a ser tratada, considerando representações 


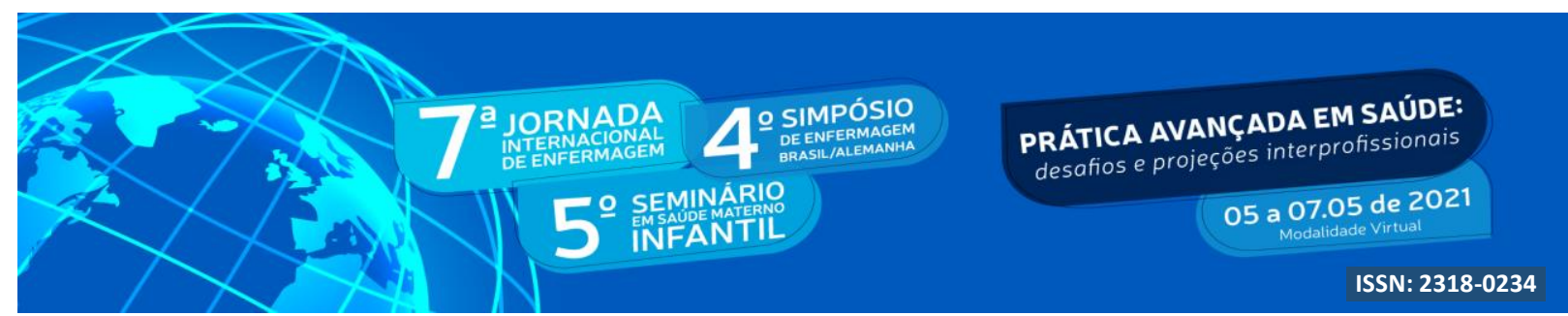

ou interpretações do mundo (MINAYO, 2013).

Em relação a abordagem do tipo qualitativo, conforme Ludke e André (2013), configura-se uma coleta direta de dados, na qual o pesquisador é próprio instrumento e os dados coletados são preferencialmente descritivos, buscando assim, compreender fatos nos cenários naturais, interpretar a experiência humana e o valor atribuído pelos atores que a vivenciam.

Realizou-se a pesquisa, no período de março de 2021, por acadêmicas de enfermagem. Esta, iniciou a partir de uma busca na Biblioteca Virtual em Saúde (BVS), utilizando as bases de dados: Literatura Latino Americana e do Caribe em Saúde (LILACS), Base de dados em Enfermagem (BDENF), Medical Literature Analysis and Retrieval System Online (MEDLINE) e Índice Bibliográfico Espanhol em Ciências da Saúde (IBECS) utilizando os descritores de assunto: "TESTE DE GUTHRIE" OR “TESTE DO PEZINHO" AND “'ENFERMAGEM". Encontrou-se 392 estudos, MEDLINE (321), LILACS (37), BDENF (25), Ibecs (12), somente 160 com textos completos na íntegra. Foram excluídos textos incompletos, línguas estrangeiras e estudos realizados em tempo superior a 10 anos, restando LILACS (12), BDENF (07), MEDLINE (2). Destes, apenas 18 obtinham textos completos. Após uma leitura minuciosa, resultou cinco estudos que foram apreciados mediante a leitura na íntegra.

\section{RESULTADOS E DISCUSSÃO}

Após a interpretação dos dados, definiu-se a articulação entre as informações coletadas e as referências teóricas com o objetivo de responder à importância da realização do Teste do Pezinho e a orientação do profissional de enfermagem aos pais ou responsáveis do RN. A análise dos resultados visou salientar as informações obtidas durante o estudo e a partir dessas, concretizou-se a interpretação para obter a resposta ao questionamento deste estudo.

Considerando que a Constituição da República Federativa do Brasil - norma de maior hierarquia no poder judiciário - assegura em seu artigo 196: "A saúde é um direito de todos e um dever do Estado." Entende-se que todos os neonatos, sem discriminação, têm direito como cidadãos de realizar o Teste do Pezinho, a fim de prevenir patologias físicas, motoras e 


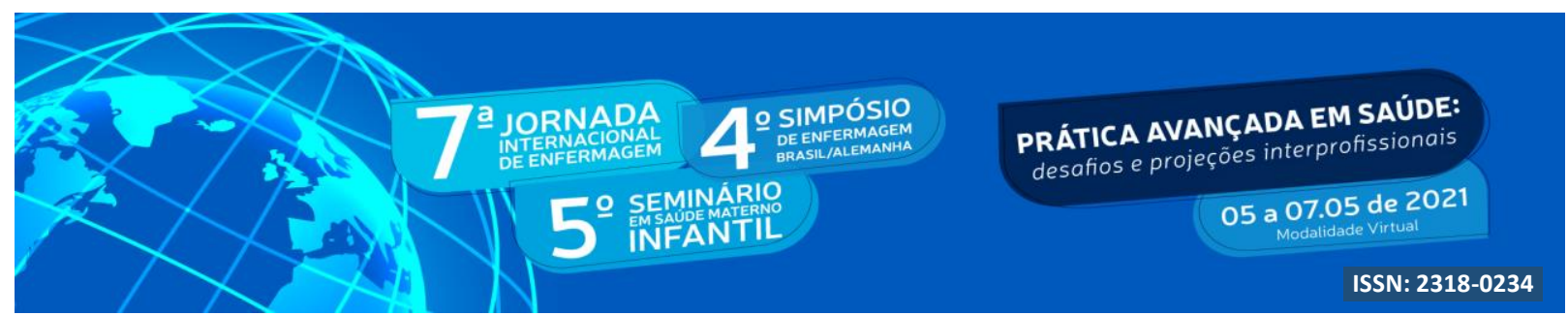

mentais, fornecidos pelo PNTN (BRASIL, 1988). A PNTN promove o acesso universal e integral no SUS, com foco na prevenção, na intervenção, no cuidado e no acompanhamento permanente das pessoas com as doenças que constam no Programa (BRASIL 2016).

Segundo Reis; Partelli (2014), os serviços de triagem neonatal, normalmente, encontram-se nas Unidades Básicas de Saúde. Atualmente, todos os estados brasileiros possuem esse serviço e podem receber habilitação para as quatro fases do exame, sendo estas: fase I consiste na detecção de hipotireoidismo congênito e fenilcetonúria. Por outro lado, a fase II, consegue diagnosticar fenilcetonúria, hipotireoidismo congênito, doenças falciformes e outras hemoglobinopatias. Ainda, a fase III, é capaz de identificar fenilcetonúria, hipotireoidismo congênito, doenças falciformes, outras hemoglobinopatias e fibrose cística. Por fim, a fase IV, detecta hiperplasia adrenal congênita e deficiência de biotinidase.

A partir da análise dos estudos incluídos evidenciou-se que em cinco anos, aproximadamente, mais de 17 mil recém-nascidos foram diagnosticados com as doenças identificadas pelo teste do pezinho. Dentre essas patologias, o Hipotireoidismo congênito e a doença falciforme correspondem $77 \%$ dos casos (BRASIL, 2019a). Conforme os dados citados anteriormente, compreende-se a relevância da elucidação e debate sobre as doenças com maior diagnóstico: o Hipotireoidismo Congênito e a Anemia Falciforme.

A Anemia Falciforme é uma anomalia na estrutura das hemácias, que assumem o formato de uma foice, ocasionando hipoxemia. Essa patologia é muito comum em lugares como a África e Oriente Médio, a qual pode afetar um a cada 50 nascimentos. No Brasil, a anemia distribui-se de forma heterogênea, devido à alta miscigenação, sendo mais comum na população negra e seus descendentes (HOCKENBERRY, Marilyn J, 2017). Corroborando, a proporção de nascidos vivos com a doença e traço falciforme/ano, pesquisas mostram que a proporção varia de acordo com o estado: Bahia 1: 650, Rio de Janeiro 1: 1.300, Pernambuco, Maranhão, Minas Gerais e Goiás 1: 1.400, São Paulo 1: 4.000, Mato Grosso do Sul 1: 5.850 e o Rio grande do Sul 1:11.000 (BRASIL, s.d.).

O (HC) configura-se uma enfermidade causada na produção dos hormônios 


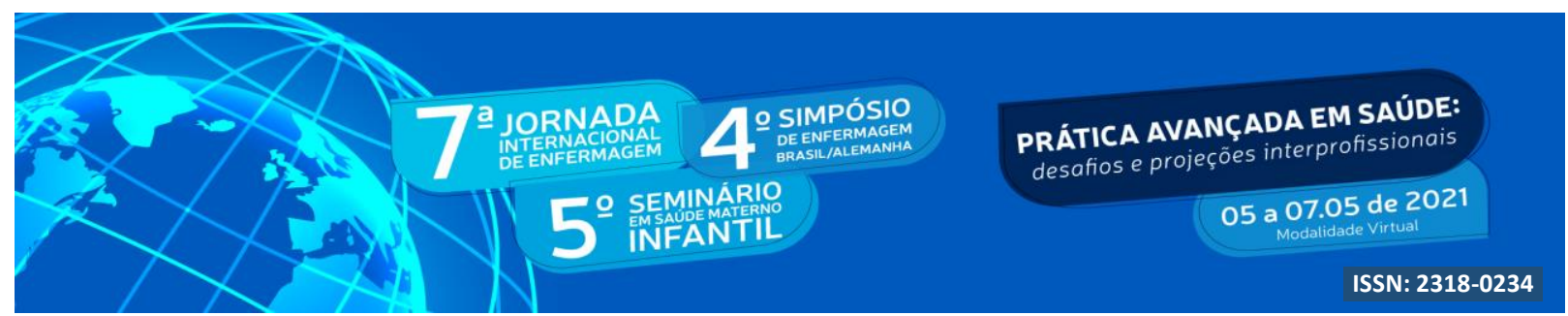

tireoidianos Triiodotironina (T3) e Tiroxina (T4). Esses hormônios são fundamentais para o funcionamento adequado do metabolismo celular, produção de energia e crescimento normal. Ainda, influencia no equilíbrio hidroeletrolítico, controla a utilização orgânica de gorduras, proteínas e carboidratos, além de promover o desenvolvimento do sistema nervoso central e a força muscular. O cretinismo biológico ocasiona o retardo no desenvolvimento físico, psicológico e sexual na criança (REIS; PARTELLI, 2014).

No Brasil, no ano de 2019, em média, 2,2 milhões de RNS foram triados através do teste no tempo considerado adequado. Desses 2,2 milhões, 59,93\% correspondem aos RN que realizaram o exame até o quinto dia de vida. Cerca de $20 \%$ efetuaram entre o sexto e oitavo dia (BRASIL, 2019). Segundo o Coordenador geral de Sangue e Hemoderivados do Ministério da Saúde, Rodolfo Firmino, as doenças quando diagnosticadas em tempo favorável, tornam-se mais fáceis de serem tratadas, evitando quadros clínicos irreversíveis. Seguindo esse pensamento, essas estatísticas demonstram a relevância da orientação adequada dos profissionais da saúde, a fim de instigar os pais ou responsáveis a realizar o teste durante o período recomendado.

Os resultados obtidos evidenciaram que as mães e os responsáveis pelo $\mathrm{RN}$ desconhecem as doenças diagnosticadas, a importância do teste e a maneira correta de realizar o exame. Isso porque, as informações e orientações dos profissionais de enfermagem são consideradas incompletas, equivocadas e muitas vezes errôneas (DELVIVO et al, 2012).

$\mathrm{Na}$ vivência profissional, tem se observado que muitos enfermeiros não têm conhecimento técnico-científico para instruir e coletar o exame. Essas lacunas estão associadas a desvalorização acerca do teste e a insegurança durante o procedimento (STREFLING et al, 2014). Ratificando, Reis e Partelli (2014) descrevem que muitos enfermeiros, técnicos e auxiliares desconhecem as doenças diagnosticadas no teste do pezinho, os procedimentos que devem ser realizados durante a coleta e o período correto para a realização do exame.

Dessa forma, fica compreensível que as desqualificações dos profissionais acarretam 


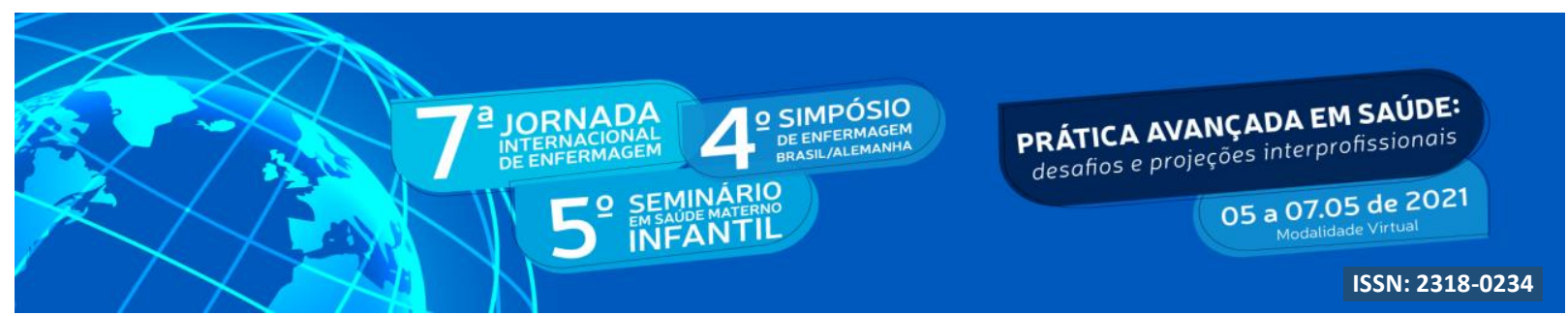

erros irreversíveis, tendo em vista que o tempo interfere no resultado. Ainda, o despreparo afeta diretamente na orientação aos pais, pois o desconhecimento torna o profissional incapaz de orientar e saciar as dúvidas referentes ao Teste de Guthrie.

Sendo assim, as observações dos estudos mostraram um conhecimento superficial sobre a Triagem Neonatal por parte dos enfermeiros. Essas omissões poderiam ser resolvidas por meio de educação continuada, com objetivo de garantir a capacitação e atualização dos profissionais para realizar os procedimentos e conscientizar a população sobre a importância da realização do teste para a prevenção de morbidades no RN (MESQUITA, et al. 2017).

Apesar do TP ser competência da equipe de enfermagem, os índices demonstram a falha da atuação dos profissionais durante o procedimento. Um estudo na cidade do Paraná, revelou que das 222.366 amostras de sangue coletadas, 2.787 delas apresentavam erros. Os equívocos mais comuns referiam-se à quantidade de sangue insuficiente, sangue envelhecido e ressecado. Diante dos resultados, constata-se que as equipes desconhecem as técnicas corretas para a realização do exame e isso exibe a desvalorização quanto a relevância da realização do procedimento (SILVA, et al. 2020). Portanto, torna-se indispensável o processo de educação permanente visando a qualificação dos profissionais, por meio da realização de cursos atualizados acerca das técnicas. Ademais, a percepção e o respeito mediante equívocos garantem a busca de novas especializações e, consequentemente, uma assistência competente.

Nesse sentido, torna-se evidente a importância do conhecimento por parte da população sobre a promoção e prevenção da saúde. Para isso, os profissionais de enfermagem obtêm um papel significativo no processo de ensino-aprendizagem, orientando os responsáveis pelo $\mathrm{RN}$ sobre as doenças detectadas no TP e salientando a necessidade de realizar o tratamento precoce em caso de resultado positivo (DELVIVO et al, 2012).

Frente a isso, o enfermeiro possui um papel significativo no PNTN, uma vez que acompanha e orienta os responsáveis do RN durante o pré-natal. Ademais, as informações sobre o teste desde a primeira consulta tornam-se relevantes para a compreensão dos pais sobre a importância do exame, a realização do mesmo e as doenças diagnosticadas. Além 


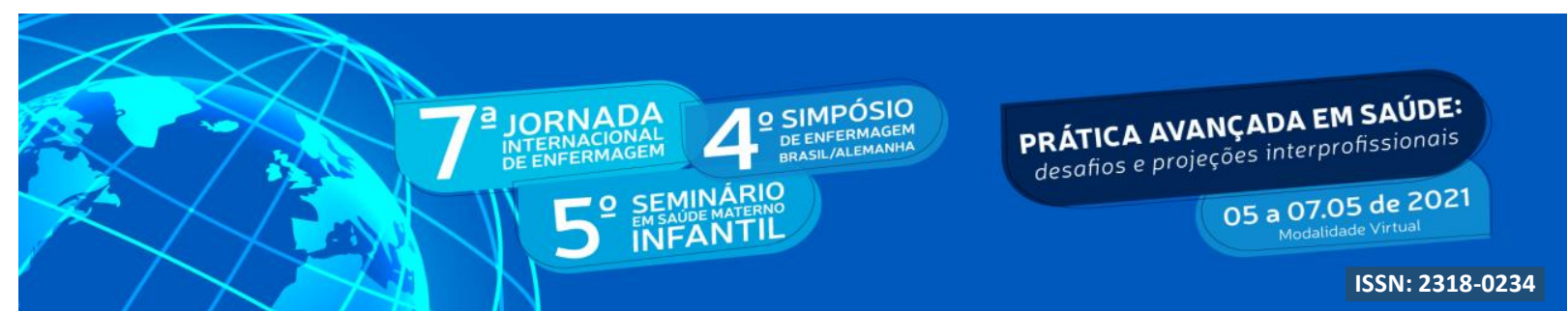

disso, o profissional deve enfatizar que o teste do pezinho é totalmente gratuito e ofertado pelo Sistema único de Saúde (OLIVEIRA, SOUZA, 2017).

Contudo, a empatia e a humanização devem ser consideradas como primordiais durante a orientação. $\mathrm{O}$ enfermeiro necessita compreender a vulnerabilidade e a aflição dos responsáveis pelo recém-nascido em relação ao exame. Para isso, cabe ao profissional estar qualificado para instruí-los corretamente sobre as etapas dos procedimentos e orientá-los sobre a relevância do exame para a saúde e qualidade de vida da criança. Além disso, convém salientar que o teste é realizado no calcanhar por se tratar de uma área de pouca sensibilidade para o RN, ou seja, a sensação de dor é mínima ou nula. Apesar de dominar o conhecimento, a equipe deve estar preparada para confortar os pais do RN se diagnosticada alguma doença. Caso isso ocorra, os enfermeiros necessitam promover uma escuta humanizada e especializada priorizando a ética. Assim, o vínculo com a família somado à confiança depositada no profissional favorece o engajamento dos familiares em realizar as recomendações necessárias.

\section{CONCLUSÃO}

Esta pesquisa permitiu salientar o quanto os profissionais de saúde necessitam refletir sobre as condutas, orientações de promoção e prevenção da saúde direcionadas aos pais e responsáveis do RN. Embora esse tema seja considerado relevante, ainda existem dificuldades relacionadas ao despreparo das equipes de saúde que abordem essa temática.

Além disso, o Teste do Pezinho mostra-se fundamental para a Triagem Neonatal, haja vista à ampla lista de doenças que abrange e o quanto o diagnóstico e tratamento precoces permitem um bom prognóstico, já que estas não possuem cura. Nesse sentido, configura-se relevante a formação permanente dos profissionais, a fim de realizar a assistência adequada, explanando a fundamentalidade de todos os exames que compõem a TN.

\section{REFERÊNCIAS}




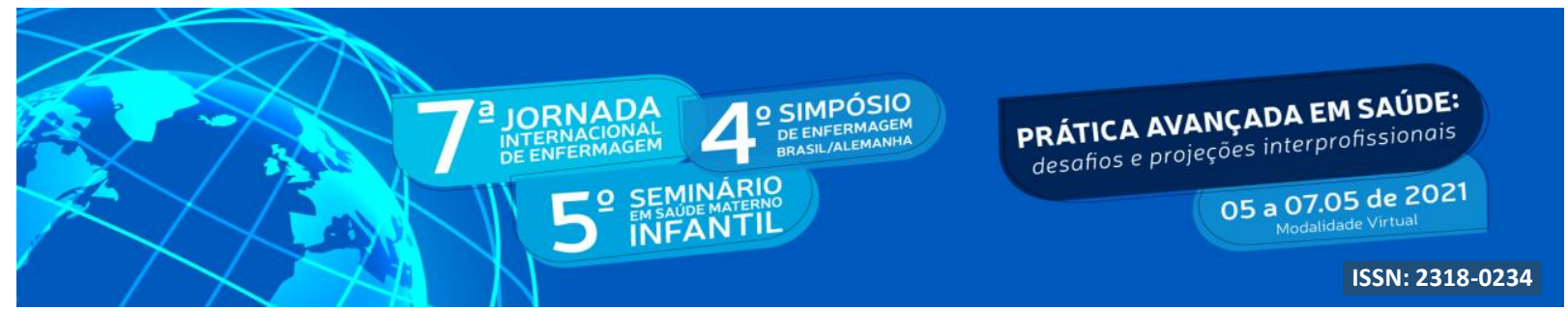

BRASIL. Constituição Federal: Art. 196º 1988. Disponível em: https://www2.senado .leg.br/bdsf/ bitstream/handle/id/518231/CF88_Livro_EC91_2016.pdf .

BRASIL. Ministério da Saúde. Biblioteca Virtual em Saúde. 06/6 Dia Nacional do Teste do Pezinho. 06 de junho de 2019. Página inicial: Ministério da Saúde, 2019a. Disponível em: <http://bvsms.saude.gov.br/ultimas-noticias/2979-06-6-dia-nacional-do-teste-do-pezinho-3>.

DA SILVA, Bruna Maciel Ribeiro et al. Atuação de enfermagem frente a coleta do teste do pezinho. revisão sistemática da literatura. Brazilian Journal of Health Review, v. 3, n. 6, p. 19087-19097, 2020. Disponível em: file:///C:/Users/Micro\%20News/Downloads/2193356310-1-PB.pdf. Acesso em: 08 mar. 2021.

DELVIVO, Elaine Migue et al. Teste do pezinho: desvelando o conhecimento das mães sobre o exame. HU Revista, Juiz de Fora, v. 38, n. 1, 2012 Disponível em: https://docs.bvsalud .org/biblioref/2016/09/2022/1944-10649-1-pb.pdf. Acesso em: 08 mar. 2021.

GIL, A. C. Como elaborar projetos de pesquisa. 6. ed. São Paulo: Atlas, 2017.) J. Genética Médica . Grupo GEN, 2017. 9788595151659 . Disponível em: https://integrada. minhabiblioteca.com.br/\#/books/9788595151659/. Acesso em: 11 de mar. de 2021

LÜDKE, M.; ANDRE, M. E. D. A. A Pesquisa em educação: abordagens qualitativas. 2 ed., E.P.U., Rio de Janeiro, 2013. Acesso em: 11 de mar. De 2021.

MARQUI, A. B. T. Teste do Pezinho e o papel da enfermagem: uma reflexão. Revista de Enfermagem e Atenção à Saúde, Minas Gerais, Brasil, v. 5, n. 2, p. 96-103, 1 jul. 2016. Disponível em: http://seer.uftm.edu.br/revistaeletronica/index.php/enfer/article/view/1605.

MESQUITA, Ana Paula Hasimoto Ribeiro et al. Profissionais de Unidades Básicas de Saúde sobre a triagem neonatal. Revista de Ciências Médicas, v. 26, n. 1, p. 1-7, 2017. Disponível em: file:///C:/Users/Micro\%20News/Downloads/3668-12863-2-PB.pdf.

MINAYO, M.C.S. O desafio do conhecimento: Pesquisa qualitativa em saúde. São Paulo: Hucitec, 2013. Acesso em: 10 de mar. de 2021.

Ministério da Saúde. Manual técnico. Secretaria de Atenção à Saúde. Departamento de Atenção Especializada e Temática. Triagem Neonatal Biológica. Brasília,DF, 2016.

Disponível em: <https://bvsms.saude.gov.br/bvs/publicacoes/triagem_neonatal_biologica _manual_tecnico.pdf $>$. Acesso em: 11 de mar. de 2021

. Ministério da Saúde. Blog da Saúde: página inicial. Conheça as doenças

diagnosticadas no Teste do Pezinho. 05 de junho de 2018. Blog da Saúde: Ministério da Saúde, 2018. Disponível em: <http://www.blog.saude.gov.br/index.php/entenda-o-sus/510786-de-junho-dia-nacional-do-teste-do-pezinho>. Acesso em: 09 mar. 2021.

Ministério da Saúde. Governo Federal: agência saúde. Mais de 2,2 milhões de 


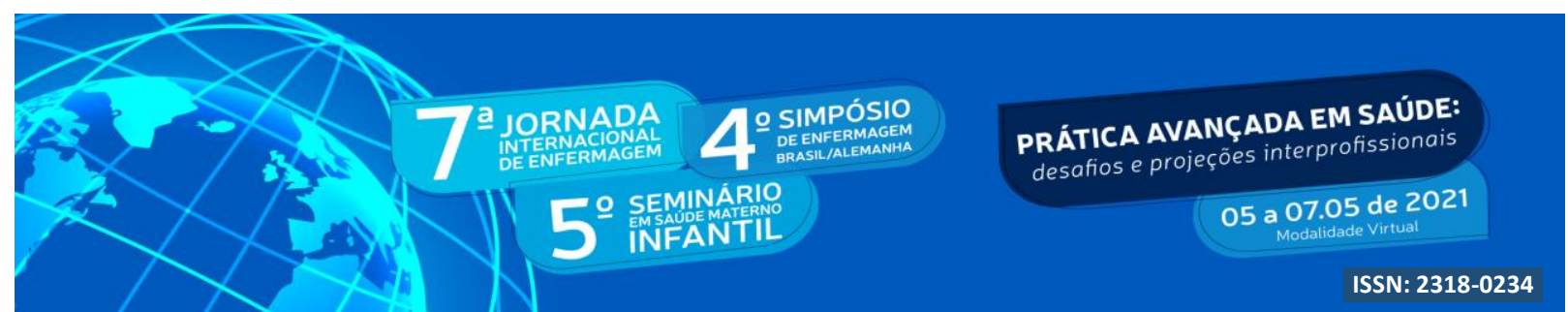

recém-nascidos fizeram o teste em 2019. 08 de junho de 2020. Brasília, DF: Ministério da Saúde, 2020. Disponível em: <https://www.gov.br/saude/pt-br/assuntos/noticias/mais-de-2-2milhoes-de-recem-nascidos-fizeram-o-teste-em-2020>. Acesso em: 10 mar. 2021.

Ministério da Saúde. Governo Federal: agência saúde. Ministério da Saúde reforça a importância do Teste do Pezinho entre o $3^{\circ}$ e $5^{\circ}$ dia de vida. 06 de junho de 2019. Brasília, DF: Ministério da Saúde, 2019b. Disponível em: <https://www.gov.br/saude/ pt-br/assuntos/noticias/ministerio-da-saude-reforca-a-importancia-do-teste-do-pezinho-entreo-3-e-5-dia-de-vida>. Acesso em: 08 mar. 2021.

. Ministério da Saúde. Secretaria de Assistência à Saúde. Coordenação-Geral de Atenção Especializada. Manual de Normas Técnicas e Rotinas Operacionais do Programa Nacional de Triagem Neonatal. Brasília, DF: Ministério da Saúde, 2002.

OLIVEIRA, Eva F.; SOUZA, Anderson P. A Importância da Realização Precoce do Teste do Pezinho: O Papel do Enfermeiro na Orientação da Triagem Neonatal. Id On Line Revista Multidisciplinar e de Psicologia, Maio de 2017, VOL.11, N.35, P. 361-378. ISSN: 1981-1179. Disponível em: <file:///C:/Users/Micro\%20News/Downloads/742-2334-1PB\%20(2).pdf>. Acesso em: 08 mar. 2021.

REIS, Elisama Ferraz Sousa; PARTELLI, Adriana Nunes Moraes. Teste do Pezinho: conhecimento e atitude dos profissionais de enfermagem. Revista Brasileira de Pesquisa em Saúde/Brazilian Journal of Health Research, 2014. Disponível em: <https://periodicos.ufes.br/rbps/article/view/8489/5985> Acesso em: 08 mar. 2021.

STREFLING, Ivanete Silva Santiago et al. Conhecimento sobre triagem neonatal e sua operacionalização. Cogitare Enfermagem, v. 19, n. 1, 2014. Disponível em: <http://www.revenf.bvs.br/pdf/ce/v19n1/04.pdf>. Acesso em: 08 mar. 2021. 